\title{
Melatonin and Myoinositol: A Foreword Step Toward IVF Success, a Prospective Clinical Trial
}

\author{
Atyaf Hasan $^{1}$, Al-Quraan Elena ${ }^{2}$, Al-Quraan Ghassan ${ }^{1, ~ *, ~ T a y s e e r ~ R i m a w i ~}{ }^{1}$, Said Nasrallah ${ }^{1}$ \\ ${ }^{1}$ Department of Iraqi Speciality Center for In-Vitro Fertilization, Baghdad, Iraq \\ ${ }^{2}$ Department of Family Medicine, Faculty of Medicine, Princess Basma Teaching Hospital, Irbid, Jordan
}

Email address:

quraangh@yahoo.com (Al-Quraan G.)

${ }^{*}$ Corresponding author

To cite this article:

Atyaf Hasan, Al-Quraan Elena, Al-Quraan Ghassan, Tayseer Rimawi, Said Nasrallah. Melatonin and Myoinositol: A Foreword Step Toward IVF Success, a Prospective Clinical Trial. Journal of Gynecology and Obstetrics. Vol. 7, No. 1, 2019, pp. 1-7.

doi: 10.11648/j.jgo.20190701.11

Received: December 2, 2018; Accepted: January 25, 2019; Published: February 19, 2019

\begin{abstract}
Many subfertile couples, who failed to conceive naturally, seek help by means of artificial reproduction techniques such as in vitro fertilization (IVF) to achieve pregnancy. It is clear that oocyte and embryo quality depression are widely considered to be the main challenge of IVF in assisted reproduction. The aim of the study was to assess the effect and the role of melatonin combined with myoinositol on oocyte quality and to investigate its correlation with pregnancy outcome. A total of 315 patient, aged between 30-40, in a 12 month study period from Jan. 2017 to Dec. 2017, with history of one or multiple unsuccessful IVF cycles due to poor oocyte quality were included in this study. The study group (group 1, n=166) was treated with melatonin combined with myoinositol (Seidivid plus, SEIDLAB) for 3 months prior to a new IVF cycle and the control group (group 2, $\mathrm{n}=149$ ) just received a standard IVF cycle without melatonin and myoinositol. IVF outcomes were compared between the two groups. Primary endpoint was the number of morphologically mature oocytes retrieved (MII oocytes). Secondary endpoints were fertilization rate per number of mature oocytes, embryo quality and pregnancy rate. After treatment, the number of mature oocytes, the fertilization rate, the number of top-quality embryos transferred and pregnancy rate were statistically higher compared to the previous IVF cycle, while there was no difference in the number of retrieved oocyte. The mean number of oocytes retrieved differ between the two groups being (11.56) in group 1 and (10.9) in control one (1920 vs. 1629) and the percentage of mature oocytes was significantly higher in melatonin-treated group (77.03\% vs. $69.1 \%)$. Concurrently, the mean number of immature oocytes (germinal vesicles) was reduced (22.9\% vs. $39.9 \%)$. Furthermore, the melatonin -myoinositol treated group showed an increase in fertilization rate $(80.2 \%$ vs. $77.7 \%)$ and the mean number of grade one embryos resulted was also higher $(60.05 \%$ vs. $49.5 \%)$. Clinical pregnancy rate was in tendency higher in the group treated with melatonin and myoinositol (48.3\% vs. $40.8 \%$ ), In the current study It was concluded that Melatonin and myoinositol treatment is likely to become a significant option for improving oocyte and embryo quality in women who cannot become pregnant because of poor oocyte quality.
\end{abstract}

Keywords: Melatonin, Myoinositol, IVF Cycle, Intracytoplasmic Sperm Injection, Oocyte Quality, Pregnancy Outcome

\section{Introduction}

The growing interest by physicians to many infertile couples, who failed to conceive due to poor oocyte quality, the great advances in assisted reproductive technologies and the extensive experience gained over this long period had shed light on parameters determining ART outcomes. It is clear that oocyte and embryo quality depression are widely considered to be the main challenge of IVF in assisted reproduction. Many studies have already investigated and proposed potential predictive factors for negative and positive IVF-ET outcomes [1, 2] and highlight the importance of many predictive factors essential for optimal oocyte maturation: the follicular fluid composition, in particular, high concentration of two different compounds such as myoinositol and melatonin, is a predictor of ART positive outcome in oocyte maturation, while the imbalance 
of reactive oxygen species had a negative impact. In humans, the negative impact of oxidative stress has been demonstrated by several studies on the number and quality of retrieved oocytes and embryos following in-vitro fertilization. More recently, it has been discovered that an imbalance of reactive oxygen species (ROS), or 'oxidative stress', can have a negative impact on the success of infertility treatments, and furthermore, investigators have begun addressing potential mechanisms of preventing these effects with the use of novel oxygen scavengers such as melatonin, a pineal hormone that regulates circadian rhythms, which has also been shown to exhibit a unique oxygen scavenging abilities. Ovarian stimulation protocols in assisted reproduction are associated with significant changes to the in-vivo follicular environment, altering endogenous levels of oxygen scavengers [3]. Furthermore, in-vitro, these oocytes are no longer protected by antioxidant-rich follicular fluid, leaving them more susceptible to oxidative damage [4-6]. They may also be exposed to high oxygen concentrations in incubators and during handling throughout the IVF process, with higher concentrations of oxygen being associated with more ROS.

The quality of oocytes plays a pivotal role in the development of a clinical pregnancy, it considered one of the main cause of fertilization failure in assisted reproductive techniques [7]. In fact oocytes of poor quality may be the cause of women infertility and an important obstacle in successful in vitro fertilization [8]. Poor oocyte quality remains a serious problem for female infertility, said Professor Hiroshi Tamura from the Yamaguchi University Graduate School of Medicine, Japan.

Several factors can affect oocyte quality and therefore pregnancy outcome in assisted reproductive technology cycles. Indeed, assisted reproductive technology techniques nowadays focus on obtaining high quality rather than high numbers of oocytes and embryos, i.e. from quantity to quality [9]. Among the factors affecting oocyte quality is follicular fluid. Recent studies highlight the important of the follicular fluid in oocyte maturation: indeed, follicular fluid composition is a predictor of ART positive outcome [10]. It provides a very important microenvironment for the development of oocytes and may be regarded as a biological "window" reflecting metabolic and hormonal processes occurring in the microenvironment of the maturing oocyte before ovulation and also as a predictor of outcome parameters such as fertilization, embryo cleavage and pregnancy rates in IVF [10]. It is reasonable to think that some biochemical characteristics of the fluid surrounding the oocyte may play a critical role in determining oocyte quality and the subsequent potential to achieve fertilization and embryo development. Recently, a number of studies have shown that the presence of several compounds in the follicular fluid positively correlates with oocyte quality and maturation, In particular, high concentration of two different compounds such as myoinositol and melatonin was shown to be essential for optimal oocyte maturation. It may be that these agents have a positive effect on pregnancy success rates following IVF treatment.

\section{Materials and Methods}

All patients, in the in vitro fertilization (IVF) Department, in a 12 month study period from Jan. 2017 to Dec. 2017, with history of one or multiple unsuccessful IVF cycles due to poor oocyte quality were considered eligible for the study. Exclusion criteria is history of failed trial for causes other than poor oocyte quality. Patients undergoing preimplantation genetic diagnosis (PGD) are also excluded since PGD patients usually are not infertile. A total of 315 patient, aged between 30-40 years, were included in this study and were randomized into two groups. The study group (group $1, \mathrm{n}=166$ ) was treated with melatonin combined with myoinositol (Seidivid plus, SEIDLAB) for 3 months prior to a new IVF cycle and the control group (group 2, $\mathrm{n}=149$ ) just received a standard IVF cycle without melatonin and myoinositol. The Human Investigation Committee at Al-Jadriah private Hospital in Baghdad approved this study and all patient gave a written informed consent before entering in the study.

All patients underwent controlled ovarian stimulation with gonadotropins (FSH urinary or recombinant), Pituitary suppression was routinely achieved with GnRH antagonist, all patients were given HCG (5000-10,000 IU) or GnRH agonist for final oocyte maturation, followed approximately 35 hours later by oocyte retrieval (Cook oocyte aspiration needle, double lumen). Cumulus and corona radiata cells were immediately removed after retrieval by a short exposure to hyaluronidase enzyme and mechanically cleaned from the remaining surrounding cumulus cells using a denuding pipette by gentle aspiration in and out of a Pasteur pipette. The denuded oocytes were then assessed for their meiotic maturation status. In preparation for ICSI, oocytes with an extruded first polar body presumably at the metaphase II stage (MII) were selected. Oocytes were inseminated by intracytoplasmic sperm injection (ICSI), and resulting embryos were cultured for 3-5 days, depending on their morphological score on Day 2 (number of blastomeres and the degree of fragmentation), in non-sequential media (LifeGlobal Media). The ICSI and blastocyst transfer were standards of care for all patients throughout the study period. In all centers the protocol for embryo transfer was the same.

\section{Results}

During the study period, 315 patients matching the inclusion criteria were randomized into two groups as previously described. Patients assigned to the study group (group 1, $\mathrm{n}=166$ ) were treated with melatonin combined with myoinositol (Seidivid plus, SEIDLAB) for 3 months prior to a new IVF cycle and Patients assigned to control group (group 2, $\mathrm{n}=149$ ) just received a standard IVF cycle without melatonin and myoinositol. No differences were found between the two groups in mean age, Body Mass Index (BMI) and duration of infertility (Table 1). The causes of infertility also didn't differ after randomization between the two groups. The ICSI procedure was indicated after evaluation of two different sperm semen samples of the male partner. 
Table 1. Characteristics and outcome of patients who received melatonin, myoinositol plus folic acid (group 1; $n=166$ ) or folic acid alone (group 2; $n=149$ ).

\begin{tabular}{llll}
\hline Variable & Group 1 & Group 2 & P value \\
\hline No. of patients & 166 & 149 & - \\
Age (yrs) & $36.3 \pm 2.4$ & $35.5 \pm 2.5$ & NS \\
Duration of infertility (months) & $46.2 \pm 18.5$ & $37.8 \pm 9.6$ & NS \\
Body mass index $\left(\mathrm{kg} / \mathrm{m}^{2}\right)$ & $26.8 \pm 7.5$ & $26.4 \pm 6.8$ & NS \\
Duration of stimulation (days) & $11.4 \pm 0.9$ & $12.4 \pm 1.4$ & .002 \\
\hline
\end{tabular}

The Total number of stimulation days were significantly reduced in the group 1 co treated with melatonin and myoinositol $(11.4 \pm 0.9$ vs. $12.4 \pm 1.3, P<0.05)$ and so the embryo transfer cancellation rate for ovarian hyperstimulation syndrome and other causes (16.8\% vs. $24.1 \%$ ). The mean number of oocytes retrieved did not differ compared to the previous IVF cycle, while the number in the current study significantly differed, being 1920 (11.56) in group 1 vs. 1629 (10.9) in group $2(p<0.05$, Table 2). Concurrently, the number of immature oocytes retrieved (germinal vesicles) and degenerated oocytes not suitable for injection was lower in the group co treated with melatonin and myoinositol compared to control group $(22.9 \%$ vs. $39.9 \%$ ), with a trend for increased percentage of MII oocytes $(77.03 \%$ vs. $69.1 \%$ Table 2). The fertilization rate dramatically increased after melatonin treatment $(80.2 \%$ versus $77.7 \%$ ) and so the cleavage rate and the rate of good quality embryos. Meanwhile, the number of grade 1 embryos was significantly increased by melatonin and myoinositol supplementation $(60.05 \%$ vs. $49.5 \% ; p<0.01$, Table 2$)$, this will likely reflect the increase in biochemical pregnancy rate observed (62.3\% vs. $55.9 \%, P<0.01)$.

Table 2. Oocyte maturity and embryo score in patients who received melatonin, myoinositol plus folic acid (group $1 ; n=166)$ or folic acid alone (group $2 ; n=$ 149).

\begin{tabular}{|c|c|c|c|}
\hline Characteristic & Group 1 & Group 2 & $P$ value \\
\hline No. of retrieved oocytes & $1920(11.56)$ & $1629(10.9)$ & NS \\
\hline No. of MII oocytes & 1479 & 1125 & NS \\
\hline MII/total oocytes retrieved (\%) & $77.03 \%$ & $69.1 \%$ & NS \\
\hline No. of immature oocytes (GV-DEG) & $441(22.9 \%)$ & $504(39.9 \%)$ & .02 \\
\hline Fertilization rate & $1187(80.2 \%)$ & $874(77.7 \%)$ & NS \\
\hline No. of embryos transferred & 398 & 309 & NS \\
\hline Embryo score grade $1(\%)$ & $60.05 \%$ & $49.5 \%$ & NS \\
\hline Embryo score grade $3(\%)$ & $4.5 \%$ & $11.1 \%$ & NS \\
\hline No. of embryo transfer & 122 & 93 & \\
\hline No. of canceled embryo transfer (\%) & $16.8 \%$ & $24.1 \%$ & \\
\hline
\end{tabular}

Clinical pregnancy also showed a positive trend in the Melatonin and myoinositol group (48.3\% $\%$ vs. $40.8 \% P=0.06$, Table 3). A total of 76 pregnancies occurred, 59 of them were confirmed echographically; 12 evolved in spontaneous abortion and in five cases we could not link patients across the entire study period.

Table 3. Pregnancy outcome of patients who received melatonin, myoinositol plus folic acid (group 1; $n=166$ ) or folic acid alone (group 2; $n=149$ ).

\begin{tabular}{llll}
\hline Variable & Group 1 & Group 2 & P value \\
\hline No. of pregnancies & 76 & 52 & NS \\
No. biochemical pregnancies, (\%) & $62.3 \%$ & $55.9 \%$ & NS \\
No. clinical pregnancies, (\%) & $48.3 \%$ & $40.8 \%$ & NS \\
\hline
\end{tabular}

An important finding in the study was that oral melatonin plus myoinositol supplementation can have a beneficial effect on the improvement of fertilization and embryo quality and was associated with significantly improved clinical pregnancy rate and this may have occurred due to a reduction in oxidative damage.

\section{Discussion}

The most common cause of ICSI failure is the reduced embryo quality and several factors such as socialenvironmental, aging and/or pathological factors, can negatively affect it [11-14], therefore, any treatment able to improve oocyte quality should be considered a foreword step towards IVF procedures success. The main factors influencing IVF outcome are oocyte and embryo quality. Indeed, the promising way to improve IVF success rate is to improve oocyte and embryo quality. Recently, it has been shown that the two molecules, normally produced by our body, Myoinositol and Melatonin are efficient predictors for oocyte quality and IVF outcomes: high concentration of both molecules positively correlates with high oocyte quality [15, $16,17]$. In particular, several clinical trials have shown that supplementation with Myoinositol, alone or in association with melatonin, is a practical approach able to improve oocyte quality and IVF outcomes in both PCOS patients and normal subjects $[14,18,19]$. Thus, melatonin and myoinositol may become the medicine of choice for improving oocyte quality $[16,20]$.

Melatonin was first isolated in 1958 as a neuro-hormone 
mainly synthesized and secreted from the pineal gland [21]. Since its discovery, further investigation has revealed that it is also produced by several other organs. Melatonin is an indoleamine, which is synthesized from the essential amino acid, tryptophan [22]. The role of melatonin ( $\mathrm{N}$-acetyl5methoxytryptamine), a small lipophilic indoleamine [23] in human reproduction is still unknown. A large body of information suggests that melatonin and the reproductive hormones are interrelated. In humans, the only data on cyclical melatonin changes comes from women undergoing ovarian stimulation. Levels of melatonin reach nadir in the preovulatory phase and peak in the luteal phase $[24,25,26]$. Melatonin influence sex steroid production at different stages of ovarian follicular maturation [16] as melatonin receptors have been demonstrated at multiple sites in ovary and in intra follicular fluid. Interestingly, melatonin receptors have been found on granulosa cells, indicating that this may be an additional site of melatonin activity [27, 28, 29].

Melatonin is normally synthesized by the ovary, as melatonin synthesizing enzymes, which present in ovarian tissue [29]. Synthesized melatonin by the ovary, may be released into the follicular fluid. However, the bulk of melatonin detected in the ovary and preovulatory follicular fluid is derived from the circulation [16]. Thus, the normally the increase in follicular melatonin concentration in the growing follicle could be an important factor in avoiding atresia. The follicle may be rescued by melatonin and this would allow a preovulatory follicle to fully develop and provide an oocyte for fertilization [16]. High level of melatonin in the follicular fluid is essential for the follicle growth, ovulation and oocyte quality, whereas reduced follicular melatonin concentration may be responsible for anovulation and poor oocyte quality [16]. It is reasonable to think that some biochemical characteristics of the Follicular Fluid surrounding the oocyte may play a critical role in determining oocyte quality and the subsequent potential to achieve fertilization and embryo development. The follicular environment is naturally protective against oxidative damage to the oocyte [30]. A recent review concluded that oral administration of melatonin reduces intrafollicular oxidative damage and increases fertilization rates [20].

Melatonin, as well as its metabolites, are claimed to be broad -spectrum antioxidants and free radical scavengers $[31,32]$. It plays as an antioxidant and free radical scavenger which protects follicles from oxidative stress, rescuing them from atresia, leading to complete follicular maturation and ovulation. elevated melatonin in preovulatory follicles, as seen in normal women, is likely to protect granulosa cells and oocyte from free radicals that are induced during ovulation. In addition, melatonin regulates the antioxidant enzymes and antiapoptotic/proapoptotic protein gene expression [16].

It appears clear that melatonin serves a purpose in the human reproductive system, with many of its observed effects likely to be related to its ability to dampen the effects of oxidative stress on the reproductive system. The relevance of oxidative stress in ART has gained increasing attention in recent literature, in particular with regards to IVF. Free oxygen radicals are created when oxygen is utilized in metabolic processes. These radicals contain 'free' valence electrons, making them highly reactive, capable of causing injury to cells [33]. Anti-oxidative agents (oxygenscavengers) are present endogenously but can also be administered exogenously. They reduce free radicals by donating electrons to stabilize them [34].

Reactive oxygen species, which produced within the follicle especially during the ovulatory process [35], has been considered to play a critical role in the success of different ARTs [36], and it is believed that oxidative stress may be a cause of poor oocyte quality [37]. Oocyte quality begins to deteriorate immediately following ovulation, a process thought to be inflammatory [38] and through its production of cytokines and proteases is associated with an increase in ROS which can inhibit oocyte maturation [39, 40, 41]. IVF can result in exposure of oocytes and embryos to high levels of superoxide free radicals, which begins prior to oocyte retrieval [42], High levels of oxidants, as $\mathrm{H}_{2} \mathrm{O}_{2}$, has been found in fragmented embryos [43]. This oxidative stress modifies the quality of oocytes and embryos, decreasing the fertilization rate and the success of the infertility treatment $[44,45,46]$. The recognition of the association between exposure of gametes and embryos to oxidative stress and a reduction in the success rates of IVF has led investigators to assess whether these adverse effects can either be prevented or reversed, with emphasis being placed on the adjuvant use of oxygen scavengers including melatonin. Melatonin is an effective mitigator of mitochondrial DNA damage [47], likely as a result of an increase in electron transport efficiency within mitochondria, thus preventing the formation of ROS [48]. Infertility treatments are associated with significant levels of reactive oxygen species which have the potential to negatively affect the quality of oocytes and embryos. Melatonin shows promise as an adjunctive therapy in the treatment of infertility. Its unique anti-oxidative characteristics and safety profile make it an ideal potential adjuvant. In some situations melatonin may be even more effective at performing this function than specific mitochondrial antioxidants [49], and this particular characteristic may have relevance to its use in the treatment of infertility and the improvement of oocyte quality and maturity.

Another parameter taken into account in terms of oocyte quality was the concentration of myoinositol in Follicular Fluid $[15,50]$. Actually no data exist on action and effects of MI, on anovulatory women of reproductive age or on spontaneous ovulation in stimulated cycles. However myoinositol is an important constituent of follicular microenvironment, playing a determinant role in both nuclear and cytoplasmic oocyte development and a positive correlation has been found between myoinositol concentration in follicular fluid and oocyte maturity and quality. Recently, an international consensus conference, further confirmed that, during IVF program, when inositol supplement, the oocyte and embryo quality in assisted 
reproduction might be better. $[51,52]$. Indeed, it was also found that, high level of myoinositol in ovarian is crucial for improved FSH signaling, oocyte maturation and embryo development. [53]. Myoinositol is an isoform of inositol, a word that collectively refers to molecules with a similar structure, a collection of nine stereoisomers, and belongs to the vitamin B complex. It is the most abundant form of inositol in nature. In fact, Inositols are pseudovitamin compounds that are falsely said to belong to the B-complex family, and are found in most foods but in highest levels in whole grains and citrus fruits. Myoinositol, an isoform of inositol, shows the most promise as a dietary supplement for promoting female fertility, restoring insulin sensitivity in instances of resistance (type II diabetes and polycystic ovarian syndrome being the most well investigated), and for reducing anxiety as well. Due to the mixed benefits to insulin resistance and fertility, myoinositol is considered a treatment of choice for PCOS in women. Myoinositol a polyalcohol classified as insulin sensitizer, and it is the second messenger of the insulin signaling [54]. It is widely distributed in nature and is considered a precursor of the synthesis of phosphoinosides, in particular it constitutes the phosphatidylinositol signal transduction system, known to be involved in the regulation of several cellular function, including cell proliferation [55]. The presence of it in human body fluid, its role as precursor of the inositol phospholipids responsible for the generation of important intracellular signals essential for mammalian oocyte development $[56,57]$ and its higher concentration in follicular fluid containing oocytes of good quality [15] suggests that the supplementation with myoinositol in IVF techniques, could positively influence the final result of the reproduction technique. In fact, in IVF techniques supplementation by myoinositol is positively related to meiotic progression of mouse germinal vesicle oocytes, enhancing intracellular $\mathrm{Ca}^{2+}$ oscillation [50]. Indeed, in human follicular fluids, higher concentrations of myoinositol provide a marker of goodquality oocytes [15]. Besides the positive effects on reducing insulin resistance $[58,59,60]$ it has been shown that myoinositol concentration in the follicular fluid directly correlates with oocytes quality [15] and additional studies also showed that myoinositol supplementation improves oocytes quality in PCOS patients [61].

Infertility treatments are associated with significant levels of reactive oxygen species which have the potential to negatively affect the quality of oocytes and embryos, in a present study the role of melatonin and myoinositol in protecting oocytes from Reactive oxygen species damage and consequently in improving oocyte quality and pregnancy rate has been detected. Considering the proven positive effects of the supplementation of myoinositol and melatonin on the follicular fluid composition and consequently on the oocyte quality, the current study was undertaken to examine whether the addition of these drugs, with its unique anti-oxidative characteristics and safety profile, improves more oocyte quality and pregnancy rate in comparison with control group in women undergoing IVF cycles.

\section{Conclusion}

The present paper was able to demonstrate that myoinositol with melatonin supplementation has a direct positive effect on oocyte and embryo quality and both drugs can create a new ray of hope for infertile patients undergoing in vitro fertilization in whom infertility is due to poor oocyte quality. Thus, melatonin and myoinositol may become the medicine of choice for improving oocyte quality.

\section{Competing Interest}

The authors have no proprietary, financial, professional, or other personal interests of any nature in any product, service, or company. The authors alone are responsible for the content and writing of the paper.

\section{References}

[1] Pacchiarotti A. Antioxidative capacity of melatonin in follicular fluid of aged IVF patients: beneficial effects on oocytes and embryo. J Gynecol Neonatal Biol 2015; 1: 1-5.

[2] Andersen AN, Witjes H, Gordon K, et al. Predictive factors of ovarian response and clinical outcome after IVF/ICSI following a $\mathrm{rFSH} / \mathrm{GnRH}$ antagonist protocol with or without oral contraceptive pre-treatment. Hum Reprod 2011; 26: 3413-23.

[3] Palini S, Benedetti S, Tagliamonte MC, De Stefani S, Primiterra M, Polli V, Rocchi P, Catalani S, Battistelli S, Canestrari F, Bulletti C: Influence of ovarian stimulation for IVF/ICSI on the antioxidant defence system and relationship to outcome. Reprod Biomed Online 2014, 29: 65.

[4] Yoshida M, Ishigati K, Nagai T, Chikyu M, Pursel V: Glutathione concentration during maturation and after fertilisation in pig oocytes: relevance to the ability of oocytes to form male pronucleus. Biol Reprod 1993, 49: 89-94.

[5] Fatehi A, Roelen B, Colenbrander B, Schoevers E, Gadella B, Beverst M, van den Hurk R: Presence of cumulus cells during in-vitro fertilisation protects the bovine oocyte against oxidative stress and improves first cleavage but does not affect further development. Zygote 2005, 13: 177-185.

[6] Huang B, Li Z, Ai J, Zhu L, Li Y, Jin L, Zhang H: Antioxidant capacity of follicular fluid from patients undergoing in-vitro fertilisation. Int J Clin Exp Pathol 2014, 7: 2273-2282.

[7] Serhal PF, Ranieri DM, Kinis A, Marchant S, Davies M, Khadum IM. Oocyte morphology predicts outcome of intracytoplasmic sperm injection. Hum Reprod 1997; 12 (6): 1267-1270.

[8] Hourvitz A, Machtinger R, Maman E, Baum M, Dor J, Levron J. Assisted reproduction in women over 40 years of age: how old is too old? Reprod Biomed Online 2009; 19: 599-603.

[9] Macklon NS, Fauser BC. Mild stimulation in in vitro fertilization. Ann NY Acad Sci 2003; 997: 105-111.

[10] Wiener-Megnazi Z, Vardi L, Lissak A, Shnizer S, Reznick AZ, Ishai D, Lahav-Baratz S, Shiloh H, Koifman M, Dirnfeld M. Oxidative stress indices in follicular fluid as measured by the thermochemiluminescence assay correlate with outcome parameters in in vitro fertilization. Fertil Steril 2004; 82 (Suppl 3): 1171-1176. 
[11] Chattopadhayay R, Ganesh A, Samanta J, Jana SK, Chakravarty BN, Chaudhury K. Effect of follicular fluid oxidative stress on meiotic spindle formation in infertile women with polycystic ovarian syndrome. Gynecol Obstet Invest 2009; 69: 197- 202.

[12] Berker B, Kaya C, Aytac R, Satiroglu H. Homocysteine concentrations in follicular fluid are associated with poor oocyte and embryo qualities in polycystic ovary syndrome patients undergoing assisted reproduction. Hum Reprod 2009; 24: 2293-2302.

[13] Van Loendersloot LL, Van Wely M, Limpens J, Bossuyt PM, Repping S, Van der Veen F. Predictive factors in in vitro fertilization (IVF): a systematic review and meta-analysis. Hum Reprod Update 2010; 16: 577-589.

[14] Unfer V, Raffone E, Rizzo P, Buffo S. Effect of a supplementation with myo-inositol plus melatonin on oocyte quality in women who failed to conceive in previous in vitro fertilization cycles for poor oocyte quality: a prospective, longitudinal, cohort study. Gynecol Endocrinol 2011 Apr 5. [Epub ahead of print].

[15] Chiu TT, Rogers MS, Law EL, Briton-Jones CM, Cheung LP, Haines CJ. Follicular fluid and serum concentrations of myoinositol in patients undergoing IVF: relationship with oocyte quality. Hum Reprod 2002; 17: 1591-1596.

[16] Tamura H, Nakamura Y, Korkmaz A, Manchester LC, Tan DX, Sugino N, Reiter RJ. Melatonin and the ovary: physiological and pathophysiological implications. Fertil Steril 2009; 92: 328-343.

[17] Chiu TT, Rogers MS, Briton-Jones C, Haines C. Effects of myo-inositol on the in-vitro maturation and subsequent development of mouse oocytes. Hum Reprod 2003; 18: 408416.

[18] Papaleo E, Unfer V, Baillargeon JP, Fusi F, Occhi F, De Santis L. Myo-inositol may improve oocyte quality in intracytoplasmic sperm injection cycles. A prospective, controlled, randomized trial. Fertil Steril 2009; 91: 1750-1754.

[19] Rizzo P, Raffone E, Benedetto V. Effect of the treatment with myo-inositol plus folic acid plus melatonin in comparison with a treatment with myo-inositol plus folic acid on oocyte quality and pregnancy outcome in IVF cycles. A prospective, clinical trial. Eur Rev Med Pharmacol Sci 2010; 14: 555-561.

[20] Tamura H, Takasaki A, Taketani T, Tanabe M, Kizuka F, Lee $\mathrm{L}$, et al. The role of melatonin as an antioxidant in the follicle. J Ovarian Res. 2012; 5: 5. [PMCID: PMC3296634] [PubMed: 22277103].

[21] Chakravarty S, Rizvi SI: Physiological effects of melatonin: implications on human health. Biomedicine 2008, 28: 252255.

[22] Reiter R, Rosales-Corral S, Manchester L, Tan D: Peripheral reproductive organ health and melatonin: ready for prime time. Int J Mol Sci 2013, 14: 7231-7272.

[23] Carlomagno G, Nordio M, Chiu TT, Unfer V. Contribution of myo-inositol and melatonin to human reproduction. Eur $\mathrm{J}$ Obstet Gynecol Reprod Biol. 2011; 159: 267-72. [PubMed: 21835536].

[24] Turi A, Garzetti GG: The pattern of melatonin in amenorrheic women affected by sterility. Acta Eur Fertil 1993, 24: 71-74.
[25] Tang P, Chan T, Tang G, Pang S: Plasma melatonin profile and hormonal interactions in the menstrual cycles of anovulatory infertile women treated with gonadotropins. Gynecol Obstet Invest 1998, 45: 247-252.

[26] Webley GE, Hearn JP: Local production of progesterone by the corpus luteum of the marmoset monkey in response to perfusion with chorionic gonadotrophin and melatonin invivo. J Endocrinol 1987, 112: 449-457.

[27] Boczek-Leszczyk E, Juszczak M: The influence of melatonin on human reproduction. Pol Merkur Lekarski 2007, 23: 128130 .

[28] Salhab M, Dhorne-Pollet S, Auclair S, Guyader-Joly C, Brisard D, Dalbies-Tran R, Dupont J, Ponsart C, Mermillod P, Uzbekova S: In-vitro maturation of oocytes alters gene expression and signaling pathways in bovine cumulus cells. Mol Reprod Dev 2013, 80: 166-182.

[29] Itoh M, Ishizuka B, Kuribayashi Y, Amemiya A, Sumi Y: Melatonin, its precursors, and synthesizing enzyme activities in the human ovary. Mol Hum Reprod 1999, 5: 402-408.

[30] Knapen M, Zusterzeel P, Peters W, Steegers E: Glutathione and glutathione-related enzymes in reproduction: a review. Eur J Obstet Gynecol Reprod Biol 1999, 82: 171-184.

[31] Reiter R J, Tan DX, Maldonado MD. Melatonin as an antioxidant: Physiology versus pharmacology. J Pineal Res. 2005; 39: 215-6. [PubMed: 16098101].

[32] Tan DX, Reiter RJ, Manchester LC, Yan MT, El-Sawi M, Sainz RM, et al. Chemical and physical properties and potential mechanism: Melatonin as a broad spectrum antioxidant and free radical scavenger. Curr Top Med Chem. 2002; 2: 181-97. [PubMed: 11899100].

[33] Kojo S: Vitamin C: basic metabolism and its function as an index of oxidative stress. Curr Med Chem 2004, 11: 1041-1064.

[34] Rahman K: Studies on free radicals, antioxidants, and cofactors. Clin Interv Aging 2007, 2: 219-236.

[35] Sugino N. Reactive oxygen species in ovarian physiology. Reprod Med Biol 2005; 4: 31-44.

[36] Sikka SC. Role of oxidative stress and antioxidants in andrology and assisted reproductive technology. J Androl 2004; 25: 5-18.

[37] Sugino N. Roles of reactive oxygen species in the corpus luteum. Anim Sci J 2007; 77: 556-565.

[38] Espey L: Current status of the hypothesis that mammalian ovulation is comparable to an inflammatory reaction. Biol Reprod 1994, 50: 233-238.

[39] Reiter R, Rosales-Corral S, Manchester L, Tan D: Peripheral reproductive organ health and melatonin: ready for prime time. Int J Mol Sci 2013, 14: 7231-7272.

[40] Tamura H, Takasaki A, Miwa I, Taniguchi K, Maekawa R, Asada H, Taketani T, Matsuoka A, Yamagata Y, Shimamura K, Morioka H, Ishikawa H, Reiter RJ, Sugino N: Oxidative stress impairs oocyte quality and melatonin protects oocytes from free radical damage and improves fertilisation rate. $\mathrm{J}$ Pineal Res 2008, 44 (3): 280-87.

[41] Tamura 13. Reiter R, Rosales-Corral S, Manchester L, Tan D: Peripheral reproductive organ health and melatonin: ready for prime time. Int J Mol Sci 2013, 14: 7231-7272. 
[42] Guerin P, El Mouatassim S, Menezo Y: Oxidative stress and protection against reactive oxygen species in the preimplantation embryo and its surroundings. Hum Reprod Update 2001, 7: 175-189.

[43] Yang HW, Hwang KJ, Kwon HC, Kim HS, Choi KW, Oh KS. Detection of reactive oxygen species (ROS) and apoptosis in human fragmented embryos. Hum Reprod 1998; 13: 9981002.

[44] Takahashi T, Takahashi E, Igarashi H, Tezuka N, Kurachi H: Impact of oxidative stress in aged mouse oocytes on calcium oscillations at fertilisation. Mol Reprod Dev 2003, 66: 143152.

[45] Du Plessis S, Makker K, Desai N, Agarwal A: Impact of oxidative stress on IVF. Expert Rev Obstet Gynecol 2008, 3: $539-554$.

[46] Combelles C, Gupta S, Agarwal A: Could oxidative stress influence the in vitro maturation of oocytes? Reprod Biomed Online 2009, 18: 864-880.

[47] El-Raey M, Geshi M, Somfai T, Kaneda M, Hirako M, AbdelGhaffar A, Sosa G, El-Roos M, Nagai T: Evidence of melatonin synthesis in the cumulus oocyte complexes and its role in enhancing oocyte maturation in-vitro in cattle. Mol Reprod Dev 2011, 78: 250-262.

[48] Hardeland R: Antioxidative protection by melatonin: multiplicity of mechanisms from radical detoxification to radical avoidance. Endocrine 2005, 27: 119-130.

[49] Lowes D, Webster N, Murphy M, Galley H: Antioxidants that protect mitochondria reduce interleukin- 6 and oxidative stress, improve mitochondrial function, and reduce biochemical markers of organ dysfunction in a rat model of acute sepsis. Br J Anaesth 2013, 110: 472-480.

[50] Chiu TT, Rogers MS, Briton-Jones C, Haines C. Effect of myo-inositol on the in-vitro maturation and subsequent development of mouse oocytes. Hum Reprod 2003; 18: 408416.

[51] Bevilacqua A, Carlomagno G, Gerli S, et al. Results from the International Consensus Conference on myo-inositol and Dchiroinositol in obstetrics and gynecology-assisted reproduction technology. Gynecol Endocrinol 2015; 31: 4416.
[52] Facchinetti F, Bizzarri M, Benvenga S, et al. Results from the International Consensus Conference on Myo-inositol and dchiroinositol in obstetrics and gynecology: the link between metabolic syndrome and PCOS. Eur J Obstet Gynecol Reprod Biol 2015; 195: 72-6.

[53] Chiu TTY, Rogers MS, Law ELK, et al. Follicular fluid and serum concentrations of myo-inositol in patients undergoing IVF: relationship with oocyte quality. Hum Reprod 2002; 17: 1591-6.

[54] Cohen P. The twentieth century struggle to decipher insulin signalling. Nat Rev Mol Cell Biol 2006; 7: 867-873.

[55] Berridge MJ, Irvine RF. Inositol phosphates and cell signalling. Nature 1989; 341 (6239): 197-205.

[56] Indyk HE, Woollard DC. Determination of free myo-inositol in milk and infant formula by high performance liquid chromatography. Analyst 1994; 119: 397-402.

[57] Fujiwara T, Nakada K, Shirakawa H, Miyazaki S. Development of inositol trisphosphate-induced calcium release mechanism during maturation of hamster oocytes. Dev Biol 1993; 156: 69-79.

[58] Genazzani AD, Lanzoni C, Ricchieri F, Jasonni VM. Myoinositol administration positively affects hyperinsulinemia and hormonal parameters in overweight patients with polycystic ovary syndrome. Gynecol Endocrinol 2008; 24: 139-144.

[59] Costantino D, Minozzi G, Minozzi E, GuaraldI C. Metabolic and hormonal effects of myo-inositol in women with polycystic ovary syndrome: a double blind trial. Eur Rev Med Pharmacol Sci 2009; 13: 105-110.

[60] Giordano D, Corrado F, Santamaria A, Quattrone S, Pintaudi B, DI Benedetto A, D'anna R. Effects of myo-inositol supplementation in postmenopausal women with metabolic syndrome: a perspective, randomized, placebo-controlled study. menopause 2011; 18: 102-104.

[61] Papaleo E, Unfer V, Baillargeon JP, Fusi F, Occhi F, De Santis L. Myo-inositol may improve oocyte quality in intracytoplasmic sperm injection cycles. A prospective, controlled, randomized trial. Fertil Steril 2009; 91: 1750-1754. 